\title{
Perspective
}

PERSPECTIVE Actualité en histoire de l'art

\section{2 | 2010}

\section{Antiquité/Moyen Âge}

\section{Archéologie, politique et histoire en débat}

Archaeology, politics, and history in debate

\section{Zeynep Çelik}

\section{OpenEdition}

Journals

Édition électronique

URL : http://journals.openedition.org/perspective/1127

DOI : 10.4000/perspective. 1127

ISSN : 2269-7721

Éditeur

Institut national d'histoire de l'art

Édition imprimée

Date de publication : 31 décembre 2010

Pagination : 271-276

ISSN : 1777-7852

\section{Référence électronique}

Zeynep Celik, «Archéologie, politique et histoire en débat », Perspective [En ligne], 2 | 2010, mis en ligne le 13 août 2013, consulté le 01 octobre 2020. URL : http://journals.openedition.org/perspective/1127 ; DOI : https://doi.org/10.4000/perspective. 1127 


\section{Archéologie, politique et histoire en débat}

\section{Zeynep Çelik}

\author{
- James Cuno, Who Owns Antiquity? Museums and the Battle \\ over Our Ancient Heritage, Princeton/Oxford, Princeton \\ University Press, 2008. 256 p., 6 fig. en n. et b. \\ ISBN : 978-0-691-13712-4; \$24,95 (19€). \\ - James Cuno éd., Whose Culture? The Promise of Museums \\ and the Debate over Antiquities, Princeton/Oxford, Princeton \\ University Press, 2009. 232 p., 43 fig. en n. et b. \\ ISBN : 978-0-691-13333-1; \$24,95 (19€). \\ - James F. GoodE, Negotiating for the Past: Archaeology, Na- \\ tionalism, and Diplomacy in the Middle East, 1919-1941, Austin, \\ University of Texas Press, 2007. 308 p., 34 fig. en n. et b., \\ 4 plans. ISBN : 978-0-292-71498-4; \$22,95 (18 €). \\ - Das große Spiel. Archäologie und Politik zur Zeit des Kolonia- \\ lismus (1860-1940), Charlotte Trümpler, éd., (cat. expo., \\ Essen, Ruhr Museum, 2010), Essen, Ruhr Museum/Co- \\ logne, DuMont, 2008. 668 p., nombreuses fig. en n. et b. \\ et en coul. ISBN : 978-3-8321-9063-7; 49,90€. \\ - Yannis HamiLAKIs, The Nation and Its Ruins: Antiquity, \\ Archaeology, and National Imagination in Greece, Oxford, \\ Oxford University Press, 2007. 376 p., nombreuses fig. \\ en n. et b. ISBN : 978-0-19-923038-9; £69 (83€).
}

\section{Appel à l'universalisme}

Les récentes controverses sur la propriété des ouvres d'art antique, qui mettaient en cause des institutions aussi prestigieuses que le Metropolitan Museum of Art (New York) et le Getty Museum (Los Angeles), se sont vues accompagnées d'une déferlante de publications liées à la question de l'héritage culturel et à la légitimité des demandes de restitution. Partant des idées développées sur le meilleur moyen de préserver les objets historiques, de les rendre aussi accessibles que possible et d'enrichir la connaissance du passé, un groupe de directeurs de musées et de spécialistes a lancé une campagne visant à promouvoir l'idée d'un musée "universel "

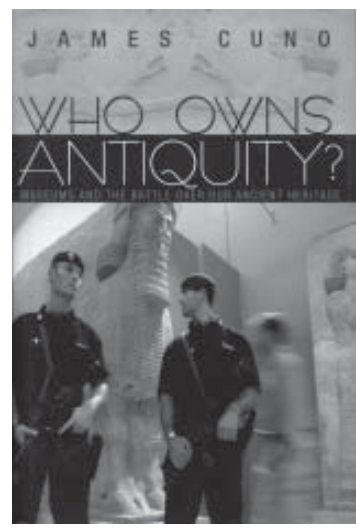

et " encyclopédique ", présenté comme "l'une des meilleures institutions du monde occidental, une invention du XIX ${ }^{\mathrm{e}}$ siècle qui a conservé un rôle essentiel dans nos vies " ${ }^{1}$, une structure qui porte en elle les aspirations au cosmopolitisme. Neil MacGregor, directeur du British Museum, affirme ainsi que les objectifs du musée londonien ont toujours été de s'adresser au monde entier au moyen d'une muséographie mettant en scène des objets de provenances diverses et de faire appel à la connaissance pour former une nouvelle sorte de citoyen mondial ${ }^{2}$.

On trouve en la personne de James Cuno un fervent défenseur de ce mouvement. Auteur de Who Owns Antiquity? Museums and the Battle over Our Ancient Heritage (CUNO, 2008) et responsable scientifique de Whose Culture? The Promise of Museums and the Debate over Antiquities (CUNO, 2009 ; fig. 1), le président et directeur de l'Art Institute de Chicago investit le «musée encyclopédique " d'une mission humaniste, le consacrant en tant que plate-forme d'exposition du patrimoine culturel mondial. Déclarant que cette idée du musée profite à tous, il demande que ses campagnes d'acquisition soient considérées d'un œil favorable. Selon lui, cette entreprise, découlant de la curiosité intellectuelle des Lumières - qui cherchaient à organiser et à classifier la culture sous toutes ses formes et à la mettre à disposition de tous - se heurte actuellement à des programmes nationalistes soutenus par des lois restrictives au regard de l'export et de la propriété. Cuno et ses confrères affirment que la situation est rendue plus difficile encore par la prise de position de la communauté internationale des archéologues en faveur des "gouvernements nationalistes ", dans

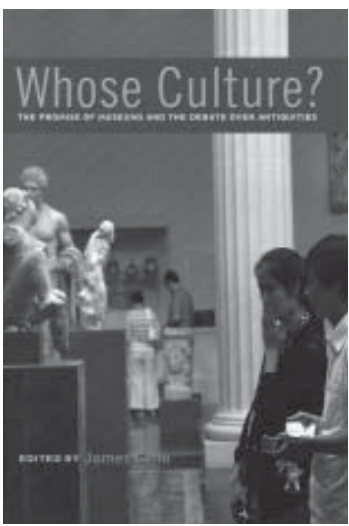
le but de protéger les sites du pillage et de la destruction. Ils lui objectent, d'une part, qu'il n'est pas nécessaire que les œuvres $\mathrm{d}$ 'art antiques soient dans leur contexte originel pour être analysées et exploitées à des fins de recherche et, d'autre 1a. CUNO, 2008, couverture ;

b. CUNO, 2009, couverture. 
part, que les objets conservés dans les musées, bien qu'étudiés de manière isolée, conduisent néanmoins à soulever de nombreuses questions d'ordre général ${ }^{3}$. Selon MacGregor, les musées encyclopédiques offrent même un contexte plus favorable et bien plus ample que les sites archéologiques eux-mêmes, permettant de mettre en regard, par exemple, des objets grecs et romains avec des œuvres chinoises (CUNO, 2009, p. 42).

L'utilisation de l'archéologie par des politiques à tendance nationaliste dans les pays " sources ", tels que la Grèce, l'Italie, l’Égypte, la Turquie, l'Iran et la Chine, est au cœur du débat. Les analyses des discours fondateurs de la nation y sont ponctuées par l'affirmation que ce n'est pas forcément le pays d'origine qui assimile, comprend et finalement possède la culture à l'intérieur de ses frontières. Selon ce courant de pensée des partisans du "musée universel ", dans notre ère de mondialisation, d'économie et de commerce internationaux, et compte tenu des régimes souvent instables et de l'incapacité de ces pays à valoriser leur héritage, il serait sage de s'en remettre à des " terres d'asile " et à $l^{\prime}$ « intendance internationale ${ }^{4}$. Ironiquement, les textes qui prétendent aborder le thème de l'héritage culturel dans une perspective ouverte et humaniste semblent être fondés sur les prémisses du paternalisme et du protectionnisme, un paradoxe souligné avec perspicacité par Yannis Hamilakis : "Qui a le droit de représenter l'universel ? Comment se fait-il que l'exposition de l'histoire globale de l'humanité, si un tel exercice est possible en des termes soi-disant neutres et dépolitisés, ne puisse être mise en scène qu'à Londres, à New York ou à Paris, et non au Caire, à Sao Paolo ou à Delhi ? " ${ }^{5}$.

La revendication de l'héritage patrimonial des "grands musées " se fonde donc sur l'usage - et le mésusage - de ce même patrimoine par les États-nations modernes. Cependant, la complexité du phénomène est évacuée par des études souvent rudimentaires et s'appuyant sur un nombre limité de sources. Les auteurs qui explorent l'instrumentalisation politique des antiquités ne font pas preuve d'une maîtrise convaincante de leur matériau ; leurs arguments, parsemés d'informations anecdotiques, ressemblent à une compilation de références aléatoires et répètent de manière abusive des clichés sensationnels (on finit par se lasser de l'association Saddam Hussein/ Nabuchodonosor). Il faut admettre que produire une évaluation critique de " La Question turque " de 1453 à nos jours en vingt pages ou traiter de "La Question chinoise " entre le IV siècle et l'époque contemporaine en trente-deux pages comme le fait Cuno dans Who Owns Antiquity? (CUNO, 2009, p. 67-87 et 88-120) relève du défi. Malheureusement, certains problèmes importants soulevés par cette littérature se perdent dans la confusion des arguments apportés, comme c'est le cas, par exemple, pour la question de l'avenir du patrimoine culturel des groupes minoritaires.

\section{Héritage et lutte de pouvoirs}

L'affirmation de Cuno selon laquelle « la propriété culturelle relève d'un paradigme d'ordre politique " et " la politique dessert la culture " trouve un écho dans les travaux d'historiens qui scrutent l'imbroglio que sont la construction de la nation et le passé historique, sans pour autant être partisans des "musées encyclopédiques " (CUNO, 2008, p. 11 ; 2009, p. 27). Dans Negotiating for the Past: Archaeology, Nationalism, and Diplomacy in the Middle East, 1919-1941, James F. Goode prend quatre études de cas, la Turquie, l'Égypte, l'Iran et l'Irak, en partant du principe discutable que ces pays auraient "partagé des expériences remarquablement similaires " et que tous ont vu la question de l'archéologie figurer « au programme des élites nationalistes de leur époque $"{ }^{6}$. La véritable force de l'ouvrage de Goode réside dans le fait qu'il recouvre plusieurs questions essentielles : la politique de l'archéologie, les rivalités entre les puissances occidentales et en particulier le rôle des Américains, qui ont bénéficié de ressources financières beaucoup plus importantes que les Européens à la suite de la Première Guerre mondiale. L'auteur montre notamment comment les archéologues, négociant - non sans heurts - avec les autorités locales, se sont métamorphosés en quasi-diplomates, et comment les diplomates sont devenus des archéologues amateurs, grâce à l'entremise de riches hommes d'affaires. Pour se référer à un cas précis, l'identité même des individus impliqués dans les transactions controversées entre le Metropolitan Museum de New York et le Musée des Antiquités 
d'Istanbul au sujet des fragments trouvés à Sardis (Anatolie Occidentale) corrobore la position de Goode : Howard Crosley Butler (professeur d'archéologie à Princeton University), Halil Edhem Bey (Directeur du Musée Impérial Ottoman à Istanbul), Stamos Papafrango (représentant grec envoyé aux États-Unis), J. P. Morgan (banquier), Valentine Macy (entrepreneur) et Mark-Lambert Bristol (haut-commissaire américain en Turquie).

Certains protagonistes clés se dégagent de l'analyse de Goode. Le plus en vue est sans doute l'égyptologue James Breasted, qui a lancé des expéditions archéologiques à travers toute dans la région dans l'Entre-deux-guerres et qui bénéficiait du soutien majeur des Rockefeller (fig. 2). Negotiating for the Past, qui montre également l'implication des intellectuels et des archéologues des pays sources, fait donc bien plus que relater une histoire à point de vue unique. Un bref aperçu du cas égyptien, par exemple, suffit pour comprendre l'engagement passionné de bien des figures influentes, dont les recherches scientifiques se faisaient l'écho de leurs affiliations politiques. Cet éventail de personnalités s'étend des poètes (Abbas Mahmud Al-Aqqad) aux journalistes (Muhammad Husayn Haykal), et des politiciens (Sa`d Zaghlul) aux romanciers (Naguib Mahfouz). Ce sont ceux qui ont relié l'histoire stratifiée de l'Égypte - des temps Pharaoniques à la période arabe - à son identité culturelle du XX $\mathrm{X}^{\mathrm{e}}$ siècle.

La dernière décennie a vu un certain nombre d'ouvrages scientifiques, souvent issus de thèses de doctorat, traiter des thèmes abordés dans Negotiating for the Past à la lumière d'un seul pays ${ }^{7}$. En revenant sur le XIX ${ }^{\mathrm{e}}$ siècle et en étudiant les débats sur la recherche archéologique, le droit d'appropriation des œuvres antiques, les liens entre la constitution d'empires et le patrimoine culturel, et les luttes des puissances politiques à l'échelle internationale, ces ouvrages permettent de placer les débats actuels sur le patrimoine dans une perspective historique. Ils dressent et balisent un panorama inestimable qui dévoile l'interaction entre l'impérialisme et le nationalisme, et mettent en lumière des relations interculturelles selon des points de vue multiples. Le plus récent de ces travaux critiques, The Nation and Its Ruins:
Antiquity, Archaeology, and National Imagination in Greece de Yannis Hamilakis (HAMILAKIs, 2007), est exemplaire par son ampleur, sa rigueur théorique et sa méticulosité, son évaluation critique des usages politiques du passé et sa prise de position retentissante contre l'idée d'un musée " encyclopédique " ou " humaniste ".

Hamilakis porte un regard sans précédent sur les vestiges helléniques - les antiquités par excellence - en étudiant le rapport existant entre archéologie, Antiquité et imaginaire national. Son cheminement va de l'introduction du néo-hellénisme en Grèce à la fin du XVIII ${ }^{\mathrm{e}}$ et au début XIX siècle, à son appropriation par les sociétés grecques locales durant la seconde moitié du XIX ${ }^{\mathrm{e}}$ siècle et jusqu'à sa cristallisation comme base permettant de forger et de reproduire les identités nationales. Invitant à une lecture de l'instauration de structures vouées à l'archéologie en Grèce (musées, instituts, écoles, etc.), Hamilakis s'interroge sur le lien qui existe entre les manifestations matérielles des civilisations antiques et l'idée de la Grande Grèce qui a perduré jusqu'en 1922 (fins de la Guerre gréco-turque), sur l'effet de l'immigration des Grecs d'Anatolie dans la reconstruction d'une identité - et d'un mythe - national ainsi que sur la guerre civile grecque, au cours de laquelle l'Antiquité classique fut utilisée comme moyen d'endoctrinement contre les "idéologies étrangères " telles que le communisme. Le cas de la rééducation des détenus du camp de concentration de l'île de Makronisos (1947-1957) en faisant appel à l'Antiquité classique est un épisode marquant d'une violence notoire : les prisonniers étaient forcés à construire des répliques de monuments helléniques, avec le Parthénon en

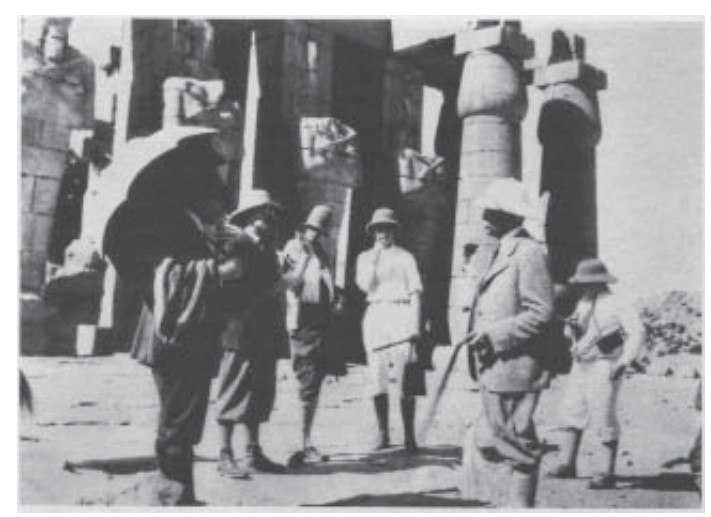

2. James Breasted avec les Rockefeller au Ramesséum à Louxor lors de leur visite en Égypte en 1929 [dans GOODE, 2007, p. 116]. 


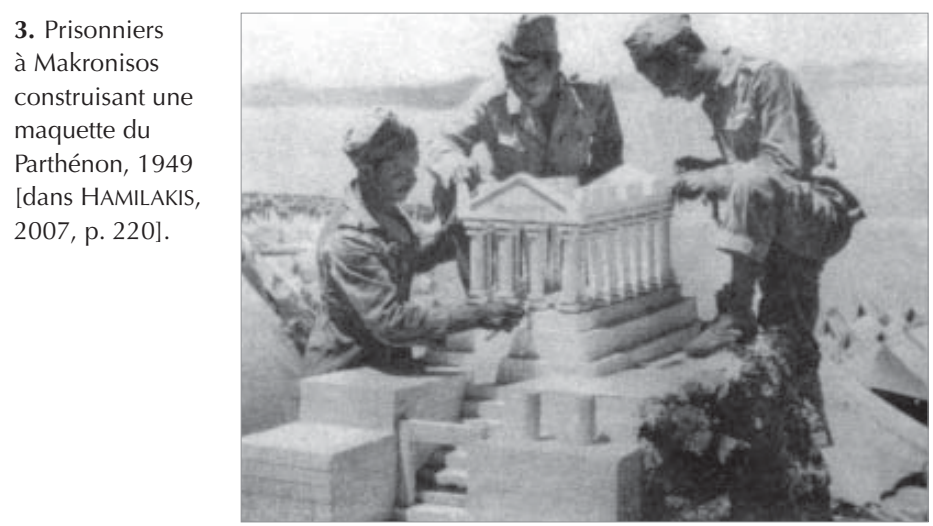

point d'orgue, dans un but d'atteindre " la plus poussée des constructions morales et le remodelage de l'individu $»^{8}$ (fig. 3).

Hamilakis situe l'héritage classique en Grèce au sein d'une appréhension complexe du nationalisme en tant que "système culturel, idéologie et ontologie, un ensemble d'idées qui définit l'existence-au-monde des individus, organise leur existence physique et sociale, leur imaginaire et même leurs rêves communautaires $"{ }^{9}$. Son analyse lucide de la relation entre nationalisme et colonialisme, ancrée dans l'archéologie, le conduit à soutenir que l'autorité morale dont on a investi les antiquités classiques a suscité des interprétations multiples et souvent contradictoires de leur pouvoir symbolique. Ce phénomène est associé à «un panoptique qui prend souvent la forme de l'Occident, et qui exige des Grecs modernes une intendance de leur passé digne de leurs ancêtres glorieux ${ }^{10}$. Pour clore sa démonstration, Hamilakis en appelle au conflit à propos des marbres Elgin, les célèbres fragments du Parthénon, qui incarnent d'une part la puissance impériale britannique et qui sont devenus d'autre part les symboles les plus connotés de l'identité nationale grecque ${ }^{11}$.

\section{Archéologie et politiques coloniales}

Les ouvrages scientifiques qui s'intéressent au lien entre archéologie, nationalisme et colonialisme à partir d'un regard critique porté sur l'histoire et qui, ce faisant, soulèvent une myriade de questions épineuses, ne sont que rarement mentionnés dans la littérature qui promeut l'idée de musées " universels " ou " encyclopédiques ». En revanche, le catalogue exhaustif de
Charlotte Trümpler, Das große Spiel. Archäologie und Politik zur Zeit des Kolonialismus (1860-1940) (Das Große Spiel..., 2008), qui accompagne l'exposition éponyme organisée au Ruhr Museum à Essen du 11 février au 13 juin 2010, intègre les débats récents tout en laissant complètement de côté les revendications de James Cuno et de ses confrères. L'objectif principal de cette manifestation et de la publication est d'explorer la politique coloniale en matière d'archéologie. En dehors des protagonistes habituels Anglais, Français et Allemands, le projet inclut Italiens, Suédois et Russes, présentant ainsi un spectre complet de personnalités, certaines bien connues, d'autres plutôt obscures, mais qui ont toutes joué un rôle essentiel. La regrettable omission de figures clés venues de l' " autre " côté, les plus notables étant Osman Hamdi Bey et Hormudz Rassam, évoqués à la volée, démontre qu'on ne leur accorde pas l'espace qu'ils méritent dans l'historiographie de l'archéologie. La publication comme l'exposition ouvrent toutefois de nouvelles perspectives en couvrant l'archéologie en l'Afrique, au Proche-Orient, en Asie Centrale et en Extrême-Orient - bien que ces différentes parties du monde ne soient pas considérées sur un pied d'égalité.

Les cinquante-neuf essais de cette publication d'ampleur (666 pages) sont divisés en treize chapitres : contexte historique, rapports militaires, organismes religieux, rivalités, acquisition, économie, pouvoir, guerre, administration, occupation, tourisme, médias et politique. Le choix des thèmes et la franchise du vocabulaire employé dépouillent l'archéologie de ses revendications à être une science neutre et l'impliquent dans les événements historiques. Les auteurs des essais, des universitaires, conservateurs ou directeurs de musée d'Europe occidentale, présentent une vision globale des pratiques européennes, la seule exception étant la contribution de Lévon Nordiguian, directeur du Musée de la Préhistoire Libanaise ${ }^{12}$. Brèves mais instructives, les contributions traitent de différentes facettes de l'interaction entre archéologie et politique. Par exemple, la section consacrée au contexte historique recouvre non seulement des thèmes généraux, tels que la lutte de pouvoir entre l'Empire ottoman et 


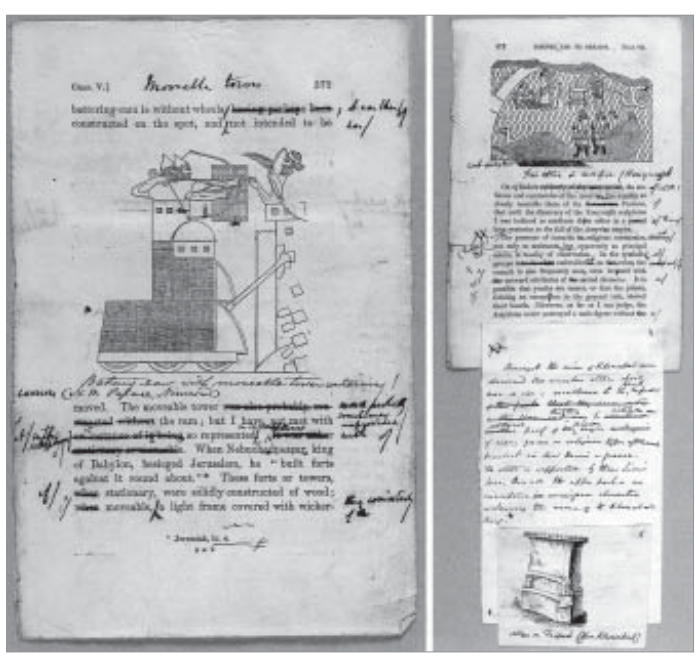

le colonialisme européen, mais aussi plus spécialisés comme la cartographie utilisée pour le recensement des sites antiques. Le chapitre " Religion " comprend des essais allant du rôle joué par l'organisme britannique Palestine Exploration Fund (PEF) à la carrière fascinante d'Alois Musil, théologien, cartographe, orientaliste, ethnographe et consultant politique, qui documenta et étudia les premiers palais islamiques en Jordanie. La section "Acquisition " s'intéresse au travail d'Austen Henry Layard en Mésopotamie en lien avec le transport des pièces assyriennes au British Museum (fig. 4), au rôle de Carl Humann pour celui de l'autel de Pergame à Berlin, à la reconstruction de la porte d'Ishtar à Berlin, ou encore aux conséquences, sur les sites archéologiques, de la concession des chemins de fer de Bagdad accordée par l'État ottoman aux Allemands. Le chapitre sur le "Pouvoir " examine la relation particulière que les empereurs Napoléon III et Guillaume II entretenaient avec l'archéologie, mettant en exergue la fascination du premier pour Jules César et les voyages du second sur les sites antiques de l'Empire ottoman au cours de sa mission pour une alliance politique forte avec le Sultan Abdülhamid II (fig. 5).

Das Große Spiel est une importante source de références. Sa bibliographie exhaustive est répartie selon six catégories : archéologie et colonialisme, tourisme, photographie et film, régions géographiques, personnalités et institutions archéologiques. Les illustrations, tirées de sources aussi diverses que des photographies et des cartes

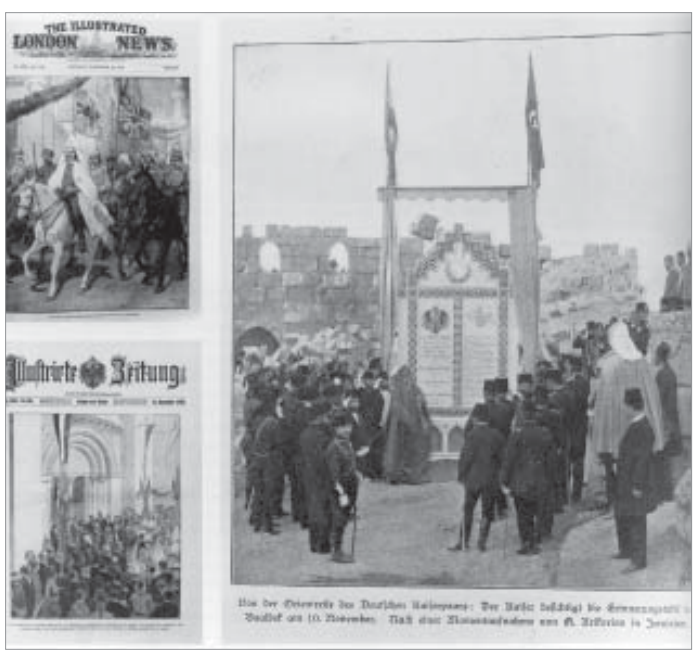

postales d'époque, des croquis originaux, des cartes anciennes, des périodiques, des livres et des peintures, se conjuguent d'une manière très riche et multidimensionnelle. De grande qualité, le catalogue sera un excellent substitut pour ceux qui n'ont pas eu la chance de voir l'exposition.

"Autres temps, autres mœurs", constate Philippe de Montebello, ancien directeur du Metropolitan, en introduction à cette question d'ordre pratique : " mais qu'en est-il du présent? " À la lumière des recherches scientifiques récentes, dont celles ici traitées, les "grands musées " trouveraient bénéfice à examiner la perspective historique dans laquelle ils se situent, plutôt qu'à adopter la politique de l'autruche en faisant passer pour vérité historique des épisodes sélectionnés par pure commodité et cherchant à justifier leur prétendu droit à s'approprier le patrimoine mondial en se cachant derrière des concepts flatteurs tels que l'humanisme et la mondialisation. Exhaustif, érudit et réfléchi, Das große Spiel... nous rappelle l'importance de mettre en regard de l'histoire les préoccupations d'aujourd'hui.

1. " ... one of the greatest institutions of the western world, first developed in the nineteenth century and still central to our lives" (CUNO, 2008, p. xxxiv-xxxv). Voir également l'introduction de Robin F. Rhodes, dans Robin F. Rhodes éd., The Acquisition and Exhibition of Classical Antiquities: Professional, Legal, and Ethical Perspectives, (colloque, Notre Dame, 2007), Notre Dame (IN), 2007, p. 7-8.
4. Épreuves de Nineveh and Its Remains, avec les corrections d'Austen Henry Layard, Édimbourg, National Library of Scotland [Das große Spiel, p. 313].

5. Articles de presse rendant compte du voyage de Kaiser Wilhelm II au Moyen-Orient en 1898 : (à gauche) son séjour à Jérusalem à I'automne 1898 (IIlustrated London News, Illustrierte Zeitung); (à droite) I'inauguration du monument à Baalbek (Liban), commémorant l'amitié entre Abdülhamid II et Kaiser Wilhelm II, le

11 novembre 1898 (Illustrierte Zeitung) [Das große Spiel, p. 402]. 
2. Neil MacGregor, "To Shape the Citizens of 'That Great City, the World' ", dans CunO, 2009, p. 39.

3. Voir, par exemple, James C. Y. Watt, "Antiquities and the Importance - or Limitations - of Archaeological Contexts ", et David I. Owen, "Censoring Knowledge: The Case for the Publication of Unprovenanced Cuneiform Tablets ", dans CUNO, 2009, p. 89-106 et 125-142.

4. Ce type d'argument est fréquent, comme on le voit avec Dennis P. Doordan, "Response to Malcolm Bell ", et Charles K. Williams II, "Response to Mary Ellen O'Connell ", dans Rhodes, 2007, cité n. 1, p. 44-45 et 114-115.

5. "Who has the right to represent the universal? Why is it that the exhibition of the global story of humanity, even if such an exercise were possible in supposedly neutral and depoliticized terms, must be staged in London, New York, or Paris, and not in Cairo, Sao Paolo, or Delhi?" (HAMILAKIS, 2007, p. 269-271). Voir aussi Yannis Hamilakis, "Response from Yannis Hamilakis ", International Journal of Cultural Property, 14/02, mai 2007, p. 160-162.

6. " shared remarkably similar experiences $[\ldots]$ on the agendas of the nationalist elites of the day " (GOODE, 2007, p. 1).

7. Sur le cas de la Turquie et la creation du Musée Impérial Ottoman d'Istanbul (1889), voir Wendy M. K. Shaw, Possessors and Possessed: Museums, Archaeology, and the Visualization of History in the Late Ottoman Empire, Berkeley/ Los Angeles/Londres, 2003. Donald Malcolm Reid, Whose Pharaohs? Archaeology, Museums, and Egyptian National Idenitity from Napoleon to World War I, Berkeley/Los Angeles/Londres, 2002, traite de l'usage de l'histoire dans l'impérialisme, de l'identité culturelle et de la construction de la nation en Égypte durant le "long " XIX siècle (1789-1914) à l'aune de l'égyptologie, des études classiques, de l'art islamique et des études coptes, en passant par les institutions de conservation en question. Frederick N. Bohrer, Orientalism and Visual Culture: Imagining Mesopotamia in Nineteenth-Century Europe, Cambridge, 2003, s'intéresse à la representation, la circulation, la réception et l'assimilation de la Mésopotamie en GrandeBretagne, en France et en Allemagne au XIX siècle. La place de l'archéologie dans le combat pour le pouvoir et la construction de la nation en Irak et la construction de l'Empire britannique est au coeur de l'ouvrage de Magnus Bernhardsson, Reclaiming a Plundered Past: Archaeology and Nation Building in Modern Irak, Austin, 2005. L'archéologie coloniale française en Algérie et le projet de relier les colonies à la métropole via l'archéologie constituent le sujet de Nabila Oulebsir, Les usages du patrimoine : monuments, musées et politique coloniale en Algérie (18301930), Paris, 2004.

8. " $[\ldots]$ the most-advanced morale-building and character re-shaping " (HAMILAKIS, 2007, p. 208).

9. " ... cultural system, ideology, and ontology, a set of ideas that define people's being-in-the-world, organize their bodily and social existence, their imagination, and even their social dreams " (HAMILAKIS, 2007, p. 15).
10. « ... panopticon that often takes the form of the west that demands from modern Greeks a stewardship of their past, worthy of their glorious ancestors " (HAMILAKIS, 2007, p. 291).

11. Le pouvoir immuable des marbres du Parthénon a refait surface avec le nouveau Musée de l'Acropole d'Athènes. Le ministre grec de la culture, Antonis Samaras, a pris position dans la controverse : "We didn't build this for the sake of the British, ... but look around: does this not negate the argument that Athens has no place good enough to house the Parthenon Marbles? ", The New York Times, 20 juin 2009.

12. "Lévon Nordiguian, Antoine Poidebard - Ein Pionier des Luftbildarchäologie ", dans Das große Spiel..., p. 85-93.

Zeynep Çelik, New Jersey Institute of Technology cel.win@ix.netcom.com 